\title{
Proceedings of the 2010 IEEE
}

International Conference on Automation and Logistics

August 16-20 2010, Hong Kong and Macau

\section{Level Set and Fat Fast Marching Method for Normal and Dynamic Path Planning of Pursuit-Evasion Problem}

\author{
Cheng-Yuan $\mathrm{Wu}$ \\ Institute of Information Science \\ Academia Sinica \\ 128 Academia Road, Section 2, Nankang, Taipei 115, Taiwan \\ cheng_yuanwu@iis.sinica.edu.tw
}

\begin{abstract}
This paper proposes a modified version of the Fast Marching Method (FMM), called Fat Fast Marching Method (FFMM), which produces a much more accurate path than the path generated by the original FMM with the approximated ODE solution. Given a complicated environment which has a long optimal path and a very large maximum cost value that results in high gradients, FFMM is faster than FMM. Furthermore, the tree structure result, a TP diagram of FFMM with path recovery rules, makes dynamic path planning of pursuit-evasion problems possible. By providing the shortest path produced by the path recovery rules in every time slot and the avoidance of re-planning in most situations, the pursuer now has a greater chance to intercept the evader in real time spectrum.
\end{abstract}

Index Terms - Level Set, Fast Marching, path planning, pursuit-evasion

\section{INTRODUCTION}

Path planning in robotic navigation has been widely researched. Its optimal aim is to find the safest and shortest path from an initial state to a goal state. A map, also known as the "configuration space," is usually used to represent the environment. Generally, this problem can be divided into four categories: On-line, Off-line, Global, and Local. Here, On-line refers to when the robot plans a path when it is still approaching the goal while Off-line refers to when a path is generated before movement. Global and Local indicate that path generation is based on either the entire map (Global) or a local sub-map (Local).

Global path planning is normally very time-consuming, since it takes all map information into account. However the result, the shortest and safest global path, is very attractive. Many methods have been proposed, such as the Voronoi diagram approach [1], cell decomposition [2] and randomized planning [3]. Although a randomized data structure, such as the rapidly exploring random tree (RRT) [3-4] performs quickly, many challenges still remain, such as how to generate sufficient samples to cover and connect the configuration space, and how to optimize the generated path. The balance between speed and optimization for global path planning should depend on the task's requirements.

Local path planning methods usually intend to find the local optimal path within a certain region. Normally, they are performed by a robot with limited range sensors or a robot with constrained computation power. We can also treat them as global path planning methods for finding a sub-goal in a sub-region. Here, examples include potential field based

\author{
Jing-Sin Liu \\ Institute of Information Science \\ Academia Sinica \\ 128 Academia Road, Section 2, Nankang, Taipei 115, Taiwan \\ liu@iis.sinica.edu.tw
}

methods [5] and fuzzy logical approaches [6]. Because of their fast performance and simplicity, they are the favorites of most real-time systems. Nevertheless, they can easily suffer from local optimal problems because only part of the entire information is provided. Some researches, such as [7], focus on avoiding the local optimal problem by considering the entire map and all possible paths. This kind of approach misleadingly turns the local problem into a global one, and using a heuristic search method or a genetic algorithm to search or train for the optimal parameters of the optimal path may require more complexity than a good global path planning method. As given an RRT without sufficient sample points, those methods cannot guarantee that the goal will be found.

On-line path planning becomes a highly valued capability for most of robot applications because nature environments are always dynamic. They intend to overcome the changes of the environment such as moving obstacles and targets. Most local path planning methods like potential filed [8] and fuzzy logical controller [9] are capable for the on-line task. One popular problem in on-line path planning is called the pursuitevasion problem. Unlike the normal path planning, the goal in the pursuit-evasion problem is dynamic which means the pursuer robot has to generate tracking path on-line in order to capture the evader. Many approaches had been studied for this problem. For example, a potential field approach had conducted in Ahmad's work [10] but it cannot guarantee the success interception for the intelligent evader and may also suffer from local optimal problem. Another approach in Warren's research [11] is trying to find the evader regions by Voronoi diagrams in the plane which allow the evader escape into them from the start position without encounter the pursuer. However, their work constrained the obstacles to be line segments.

In this paper, we provide a modified Fast Marching Method (FMM) called Fat Fast Marching Method (FFMM) to compute level sets and generate Turning points (TPs) and Turning points diagram composed by Turning point regions. Those turning points actually become the parents of the grids in their regions and make the map become a tree structure. Our method can provide the optimal path which is more accurate than the original back tracking ODE solution and may faster than the original ODE in the cases that obstacle cost value is very large or the environment is complicated which causes long optimal path solution. The piecewise result also makes real implementation much easier. Furthermore, by the result of FFMM and path update rules provided later, we 
can actually show that dynamic optimal path planning for level set methods to solve pursuit-evasion problem is possible due to the avoidance of travel time re-computation in the most situations. Our pursuit-evasion problem domain is to provide the shortest path for pursuer to capture the moving evader in every time slot within a stationary known environment. The position of the evader in every time slot is assumed to be known to the pursuer. The obstacles can be any shape without constrains.

\section{LeVel Set and Fast Marching Methods For Path PLANNING}

Level set methods proposed by Osher and Sethian[12] is an efficient numerical algorithm for solving Hamilton-Jacobi equations which are used to track the motion of interfaces propagating under complex speed laws and handle the complex topology changes. Recently, Hassouna et al. [13-15] have developed several algorithms for solving optimal path planning problems by the level set methods. The path can be very robust as it can be the shortest path, the safest path or the hybrid one.

Consider a close interface $\partial \Gamma$. Assume it monotonically moves in its normal direction with a speed function $\mathrm{s}(\mathrm{x})$ which is only increasing or decreasing and depends only on the position of the crossing point $\mathrm{x}$. The front motion can be represented as a first order non-linear PDE known as the Eikonal equation (1) with the initial condition (2).

$|\nabla T(x)| s(x)=1$

$T(\partial \Gamma)=0$

Where $T(x)$ is the travel time when the front passing through the point $x$. Fast Marching Method (FMM)[16] discussed later solves the equation with efficient one pass algorithm and the numerical approximation of $|\nabla T|$ that selects the physically correct vanishing viscosity weak $2 \mathrm{D}$ solution of (3) is given by Godunov[17].

$\max \left(D_{i j}^{-x} T_{i j},-D_{i j}^{+x} T_{i j}, 0\right)^{2}+\max \left(D_{i j}^{-y} T_{i j},-D_{i j}^{+y} T_{i j}, 0\right)^{2}=$

$1 / s_{i j}^{2}=c_{i j}^{2}$

$c_{i j}=1 / s_{i j}$

Where $D_{i j}{ }^{-} T_{i j}$ and $D_{i j}{ }^{+} T_{i j}$ are the backward and forward finite difference approximations of $T_{i j}$. Cost value function $c(x)$ is the inverse of the speed of point $x$. A large value of $c$ will be assigned to $\mathrm{x}$ if it is not feasible. When $|\nabla T|$ is approximated by first order finite differences, (3) can be rewritten to (5).

$\sum_{k=1}^{2} \max \left(\frac{T_{i j}-T_{k}}{\Delta_{k}}, 0\right)^{2}=\frac{1}{s_{i j}^{2}}$

Where $\Delta_{I}=\Delta_{x}, \Delta_{2}=\Delta_{y}, T_{1}=\min \left(T_{i-1, j}, T_{i+l, j}\right)$ and $T_{I}=\min$ $\left(T_{i, j-1}, T_{i, j+1}\right)$. The solution of (5) can be classified into the following 3 cases.

- $T_{i j}>\max \left(T_{1}, T_{2}\right): T_{i j}$ is the maximal solution of (6).

$\sum_{k=1}^{2}\left(\frac{T_{i j}-T_{k}}{\Delta_{k}}\right)^{2}=\frac{1}{s_{i j}^{2}}$
- $\quad T_{2}>T_{i j}>T_{1}: T_{i j}=T_{1}+\frac{\Delta_{1}}{s_{i j}}$

- $T_{1}>T_{i j}>T_{2}: T_{i j}=T_{2}+\frac{\Delta_{2}}{s_{i j}}$

The FMM solves (3) of each point for its arrival time by a specific order. The order is based on the relationship between points which states that the arrival time $T(x)$ of point $\mathrm{x}$ depends only on its adjacent neighbors with the smallest values. Each grid point $\mathrm{x}$ will be assigned to one of the three following sets during the front propagating process.

1. KNOWN: the computed travel time $T(x)$ of point $\mathrm{x}$ is settled and will not be changed later.

2. NARROW-BAND: the computed travel time $T(x)$ of point $\mathrm{x}$ which is the adjacent point of $K N O W N$ may be updated later.

3. FAR: the travel time $T(x)$ of point $\mathrm{x}$ is not yet computed.

Initially, all the boundary points of the front are settled to $K N O W N$ set and their neighbors are assigned to $N A R R O W$ $B A N D$ after computing their travel times by solving (3). Then, the main loop of the FMM algorithm will be recursively processed until all the points belong to KNOWN.

The steps in the loop are:

(1). For all point $x \in N A R R O W-B A N D$, compute candidate travel times by solving (3).

(2). Remove point $y \in N A R R O W-B A N D$ with the minimum travel time from $N A R R O W-B A N D$ and put point $\mathrm{y}$ into KNOWN.

(3). Add all the neighbors of point y which are not currently in KNOWN into NARROW-BAND.

(4). Repeat the loop until the travel times of all the points in the domain are settled.

Once the travel times of all points have been solved, the optimal path from the initial start point $x_{s}$ to the destination point $x_{d}$ can be back tracked by solving the following ODE equation (7).

$\frac{d x}{d t}=-\nabla T(x), x(0)=x_{d}$

Equation (7) is continually being solved until the stop criteria is meet that is $\mathrm{x}=\mathrm{x}_{\mathrm{s}}$ and $-\nabla \mathrm{T}$ shows the optimal direction to the start point. However, (4) may cause vary large gradients alone the obstacles. Therefore, the path is usually recovered by solving (8) instead of (7).

$\frac{d x}{d t}=\frac{-\nabla T(x)}{|\nabla T(x)|}, x(0)=x_{d}$

In the implementation, the ODE is solved by second order Runge-Kutta method which has the cumulative error of $O\left(h^{3}\right)$. Where $\mathrm{h}$ is the integration step. The maximal cost value $\mathrm{c}(\mathrm{x})$ of the obstacles also affects the solving speed. Large cost value slows down the path recovery speed but the value which is not large enough causes errors. The FFMM method in the next section uses the point relationship tree to recover the optimal shortest path without solving the ODE. The results of FFMM have no Runge-Kutta error and will not be affected by the maximal cost value. 


\section{FAt FASt MARChING METHOD FOR PURSUER PATH PLANNING}

\section{A. Fat Fast Marching Method for Pursuer Path Planning}

The idea behind FFMM is trying to find the piecewise optimal path composed by direct lines and turning points during the travel time computation. FFMM inherits all the idea from FMM but gains some weights by finding turning points and linking the relationships between points. This is why it is called Fat FMM. The result of FFMM is a tree structure showed in Fig. 1. Notice that every point has a parent which can only be a turning point (TP) or the initial start point $x_{s}$. All the points in the domain constitute a TP region diagram which indicates the direct parents of them. The points shared the same parent are marked in the same color showed in Fig. 9a.

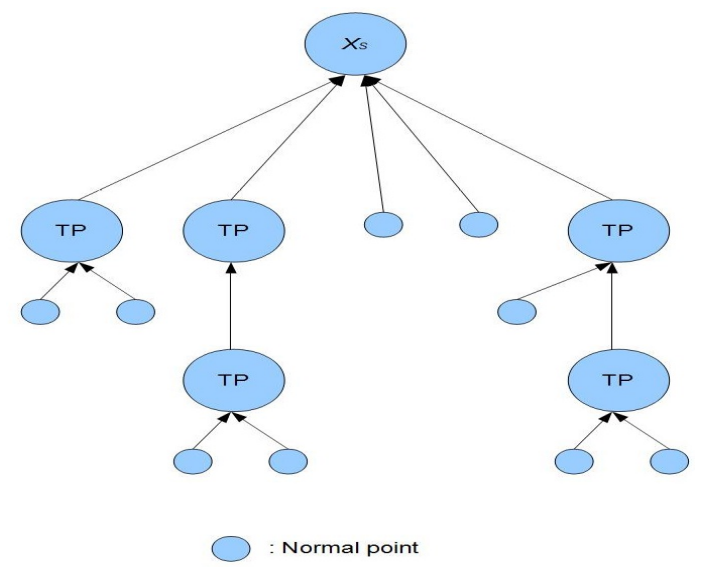

Fig. 1 The tree structure of FFMM result. Every point has a parent which can only be a turning point (TP) or the initial start point $x_{s^{*}}$

As FMM, points processed in FFMM also belong to one of the three following sets.

1. KNOWN: the computed travel time $T(x)$ and the parent Parent $(x)$ of point $\mathrm{x}$ is settled and will not be changed later.

2. NARROW-BAND: the computed travel time $T(x)$ and the parent $\operatorname{Parent}(x)$ of point $\mathrm{x}$ which is the adjacent point of $K N O W N$ may be updated later.

3. FAR: the travel time $T(x)$ and the parent Parent $(x)$ of point $\mathrm{x}$ is not yet computed.

The initial steps of FFMM is similar to FMM but with two additional steps which are setting the start point in $K N O W N$ to be a turning point and assigning the temporary parents of all the neighbors in NARROW-BAND to the start point. Then, the following loop will recursively update the travel time and the parent of each point until all the points are set in the KNOWN set.

(1). For all point $x \in N A R R O W-B A N D$, compute candidate travel times by solving (3).

(2). Remove point $Q \in N A R R O W-B A N D$ which parent is $\mathrm{P}$ with the minimum travel time from NARROW-BAND and add Q to KNOWN.

(3). Find point P's parent $\mathrm{O}$ which should be assigned already.
(4). If there exists a free line $\overline{Q O}$ that $\overline{Q O} \cap$ obstacles $=\Phi$, then assign Q's parent to point $\mathrm{O}$ that is $\operatorname{Parent}(Q)=O$.

(5). If $\overline{Q O} \cap$ obstacles $\neq \Phi$ and if there exists any TP on the line $\overline{P O}$, find the TP $\mathrm{R}$ on this line which is the closest $\mathrm{TP}$ to point $\mathrm{O}$ and $\overline{Q R}$ must be a free line. Then, assign Q's parent to point $\mathrm{R}$ that is $\operatorname{Parent}(Q)=R$.

(6). If $\overline{Q O} \cap$ obstacles $\neq \Phi$ and if there exists no TP R on the line $\overline{P O}$ that makes $\overline{Q R}$ be a free line, then set Q's parent to $\mathrm{P}$ and mark $\mathrm{P}$ as a TP.

(7). Add all the neighbors of point $\mathrm{Q}$ which are not currently in KNOWN into NARROW-BAND and assign their temporary parent to $\mathrm{Q}$.

(8). Repeat the loop until the travel times and the parents of all the points in the domain are settled.

Notice that step4 in the loop checks if Q's temporary parent $\mathrm{P}$ is a TP or not since if no obstacle between $\mathrm{Q}$ and P's parent $\mathrm{O}$, $\mathrm{P}$ is not a TP. However, there might exist a line alone the obstacle which blacks line $\overline{Q O}$. In this case, P may become a fake TP. Therefore, step5 finds the true TP $\mathrm{R}$ closest to $\mathrm{O}$ and assigns it to Q's parent. Fig. 2 gives the difference between true and fake TPs. One can compare the results in Fig. 3 with Fig. 5 and Fig. 9a to see the function of step5.

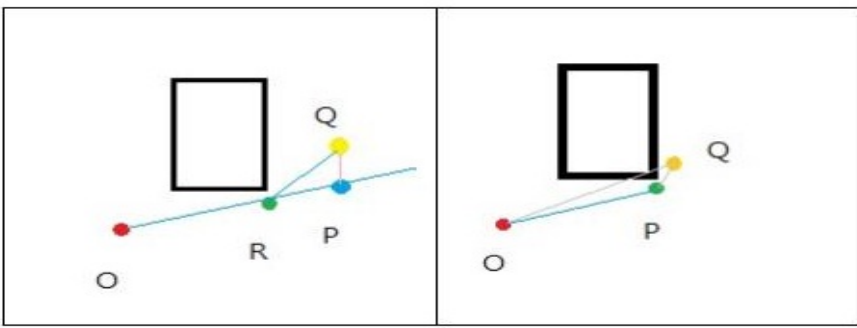

Fig. 2 The examples that $\mathrm{P}$ is a fake TP (left) and a true TP (right)

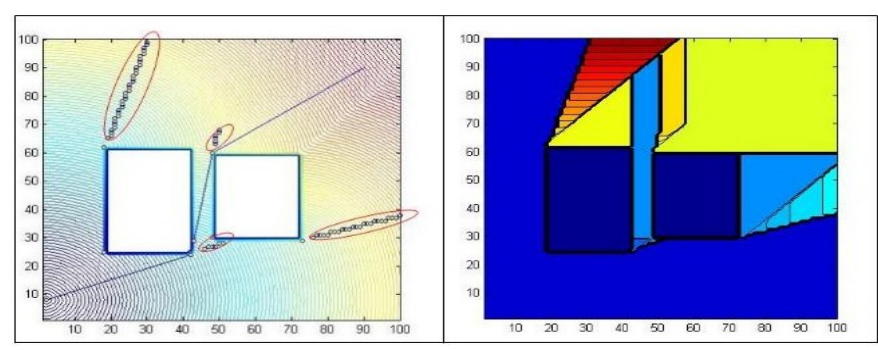

Fig. 3 Result with fake TPs and generated TP diagram.

\section{B. Online Path recovery rules}

Once the tree structure result has been generated, the optimal shortest path actually can be produced simultaneously by tracking back the parents from the destination point to the start point. Since the result is a tree structure, we can actually recover the optimal path from any point. This benefit makes the dynamic path planning available. The following path updating rules concatenate the parts of the optimal paths from the old start point to the old goal point in time $t$ and the new goal point in time $t+1$ into the new optimal path from the new start point to the new goal in time $t+1$ without redoing FFMM 
process in the most cases. For the convenience of description, we define the meanings of those following symbols and functions in the situations from time $\mathrm{t}$ to time $\mathrm{t}+1$. In time $\mathrm{t}$, we define robot's position is $S_{t}$, target's position is $D_{t}$ and the optimal path is $P_{S t 2 D t}^{\text {opt }}=\left[S_{t}, \ldots, D_{t}\right]$. In time $\mathrm{t}+1$, robot's position is $S_{t+1}$, target's position is $D_{t+1}$, the passed path is $P_{t: t+1}{ }^{\text {pass }}=\left[S_{t}, \ldots\right]$, the optimal path from $S_{t}$ to $D_{t+1}$ is $P^{\text {opt }}{ }_{S t 2 D t+1}$ $=\left[S_{t}, \ldots, D_{t+1}\right]$ and the optimal path from $S_{t+1}$ to $D_{t}$ is $P^{o p t}{ }_{S t+12 D t}$ $=\left[S_{t+1}, \ldots, D_{t}\right]=P^{o p t}{ }_{S t 2 D t}-P_{t: t+1}$ pass . Also, we define that the obstacle set is $O$, function $P(x)$ returns the parent of $x$ and function $C(x)$ returns the children set of $x$. The object of those path updating rules is to find the optimal path from $S_{t+1}$ to $D_{t+1}$ that is $P_{S t+12 D t+1}^{o p t}=\left[S_{t+1}, \ldots, D_{t+1}\right]$. The four path updating rules are:

Rule 1: If there exists a free line between $S_{t+1}$ and $D_{t+1}$ that $\overline{S_{t+1} D_{t+1}} \cap O=\Phi$, the optimal path is that line.

$\overline{S_{t+1} D_{t+1}} \cap O=\Phi \leftrightarrow P_{S_{t+1}}^{o p t} 2 D_{t+1}=\left[S_{t+1}, D_{t+1}\right]$

Rule 2: If $P\left(D_{t+1}\right)$ belongs to $P_{S_{t+1}}^{\text {opt }} 2 D_{t}$, the optimal path in time $\mathrm{t}+1$ :

$\overline{S_{t+1} D_{t+1}} \cap O \neq \Phi \wedge$

$P\left(D_{t+1}\right) \in P_{S_{t+1}}^{o p t} 2 D_{t} \leftrightarrow P_{S_{t+1}}^{o p t} 2 D_{t+1}=$

$P_{S_{t+1} 2 D_{t}}^{o p t}-\left[C\left(P\left(D_{t+1}\right)\right) \in P_{S_{t+1} 2 D_{t}}^{o p t}\right]+D_{t+1}=$

$\left[S_{t+1}, \cdots, P\left(D_{t+1}\right)\right]+D_{t+1}=\left[S_{t+1}, \cdots, P\left(D_{t+1}\right), D_{t+1}\right]$

Rule 3: If $P\left(D_{t+1}\right)$ does not belong to $P_{S_{t+1} 2 D_{t}}^{\text {opt }}$ and there exists a set $\mathrm{T}$ belonging to the intersection set of $P_{S_{t+1} 2 D_{t}}^{o p t}$ and $P_{S_{t} 2 D_{t+1}}^{o p t}$ and $S_{t}$ does not belong to $\mathrm{T}$, the optimal path in time $\mathrm{t}+1$ :

$\overline{S_{t+1} D_{t+1}} \cap O \neq \Phi \wedge P\left(D_{t+1}\right) \notin P_{S_{t+1}^{o p t} 2 D_{t}} \wedge$

$\exists T \in\left(P_{S_{t+1}{ }^{o p t}} \cap D_{t} P_{S_{t} 2 D_{t+1}}^{o p t}\right)$

$\wedge S_{t} \notin T \rightarrow P_{S_{t+1} 2 D_{t+1}}^{\text {opt }}=$

$P_{S_{t+1} 2 D_{t}}^{o p t}-\left[C(C) \in P_{S_{t+1} 2 D_{t}}^{o p t}\right]+\left[C(C) \in P_{S_{t} 2 D_{t+1}}^{o p t}\right]=$

$\left[S_{t+1}, \cdots, C\right]+\left[C, \cdots D_{t+1}\right]-C=$

$\left[S_{t+1}, \cdots, C, \cdots, D_{t+1}\right]$

Where $\mathrm{C}$ is the one of the TPs in T which is closest to $S_{t+1}$ and its children set is C(C). Fig. 4 shows one example of the path updating processed by Rule 3 .

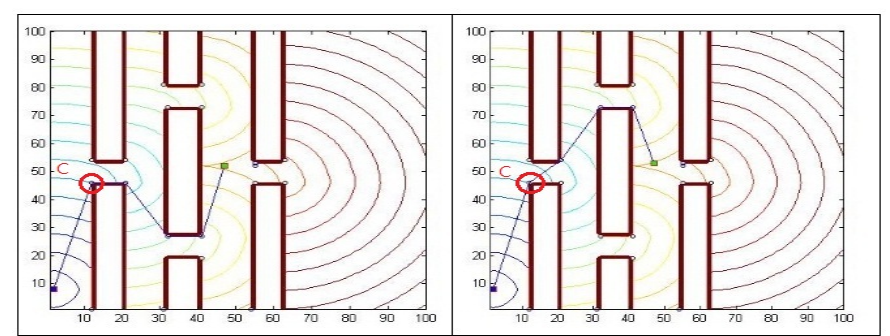

Fig. 4 The path before updated Rule 3 (left) and the path updated by Rule 3

Rule 4: If $P\left(D_{t+1}\right)$ does not belong to $P_{S_{t+1} 2 D_{t}}^{o p t}$ and there exists no set $\mathrm{T}$ belonging to the intersection set of $P_{S_{t+1} 2 D_{t}}^{o p t}$ and $P_{S_{t} 2 D_{t+1}}^{o p t}$, a new FFMM process needs to be conducted from $S_{t+1}$.

$\overline{S_{t+1} D_{t+1}} \cap O \neq \Phi \wedge P\left(D_{t+1}\right) \notin P_{S_{t+1} 2 D_{t}}^{o p} \wedge$

$\neg \exists T \in\left(P_{S_{t+1} 2 D_{t}}^{\text {opt }} \cap P_{S_{t} 2 D_{t+1}}^{o p t}\right) \rightarrow$

$\operatorname{FFMM}\left(S_{t+1}\right)$

Notice that there is no needed to use $C(x)$ to find the children set during implementations since the path is an ordered list structure and one can easily get the part of the path behind the point.

\section{DISCUSSIONS ON THE EXPERIMENT RESULTS}

Two kinds of simulations are provided here for further evaluations. We firstly compare the performances of FFMM and FMM in the normal shortest path planning problem and we then demonstrate a simulation of dynamic path planning for pursuit-evasion problem via implementing FFMM with the on-line path recovery rules. Due to the computation time concerning, all the map sizes are set to $100 \times 100$ grid points. However, one should design an adaptive grid algorithm [18] for large map problems suitable for the desired hardware to apply FMM or FFMM.

\section{A. Normal shortest path planning}

In this section, the simulations of FFMM and FFM on four maps are compared and one simulation on a complex maze of FFMM shows its capability of finding the shortest path in a complex map.

Fig. 5-8 show the shortest paths produced by FFMM and FMM. From those results we can see the paths produced by FFMM is much accurate then the paths produced by FMM especially for the vertical and horizontal lines. This is because the cumulative order 2 Runge-Kutta error affects the performance of original ODE path recovery method of FMM. Also the FFMM path is very accurate at start position but the ODE method can only approach into a certain range. However, without suitable linear interpolation error correction, some parts of the FFMM path may become slightly worse than the FMM path because one additional TP on the path may be incorrectly assigned to one TP's parent. Fig. 6 is designed here to show the result without error correction. This linear interpolation error is occurred during the obstacle blocked detection of step4 in the FFMM loop. Fortunately, 
those additional TPs are actually the necessary TPs determined by the system for bypassing the obstacles and will only cause tiny effects on the path. The result is still close to the optimal one since the error is small. One can also easily fix it by setting a good tolerance range. In Fig. 7, a typical example illustrates the well performance of FFMM on the vertical lines. Those lines are slightly twisted in the FMM path due to the rk2 error. Fig. 8 gives an example that the FMM path might get an error passing through the obstacle when the largest cost value is set not large enough but the FFMM path is not affected by the cost value. The TP diagram of map 1 in Fig. 9a shows the difference of the TP diagram in Fig. 3 after doing the step5 in the loop. We can image that we may get an error path passing through the true TP and turning at a fake TP if we waive this step. Fig. $9 \mathrm{~b}$ demonstrates that even in a very complicate maze, FFMM still has no problem to find the shortest path. TABLE. I shows when maximal cost value is set to a large number the total times of FFMM become faster than FMM in map 2-4. The speed of FFMM is stable and only depends on the scale of the map size and has no effect with the maximal cast values because its total time is almost equal to its front propagation time. Although FMM also has stable speed in propagation but its path calculation time will depend on the complexity of the environment and it's converge speed also affected by the maximal cost value.

TABLE. I

Computation times of FFMM and FMM when the largest cost value is big.

\begin{tabular}{|l|c|c|}
\hline \multicolumn{1}{|c|}{ Max cost $=50000$} & FFMM (seconds) & FMM (seconds) \\
\hline Map 1 prop Elapse & 18.025057 & 5.490282 \\
\hline Map1 Path & 0.002363 & 3.50379 \\
\hline Map1 total & 18.02742 & 8.994072 \\
\hline Map 2 prop Elapse & 17.911444 & 5.593224 \\
\hline Map 2 Path & 0.000751 & 19.264236 \\
\hline Map 2 total & 17.912195 & 24.85746 \\
\hline Map 3 prop Elapse & 17.397373 & 5.640724 \\
\hline Map 3 Path & 0.000184 & 24.171065 \\
\hline Map 3 total & 17.397557 & 29.811789 \\
\hline Map 4 prop Elapse & 17.495732 & 5.370758 \\
\hline Map 4 Path & 0.000155 & 21.749344 \\
\hline Map 4 total & 17.495887 & 27.120102 \\
\hline
\end{tabular}
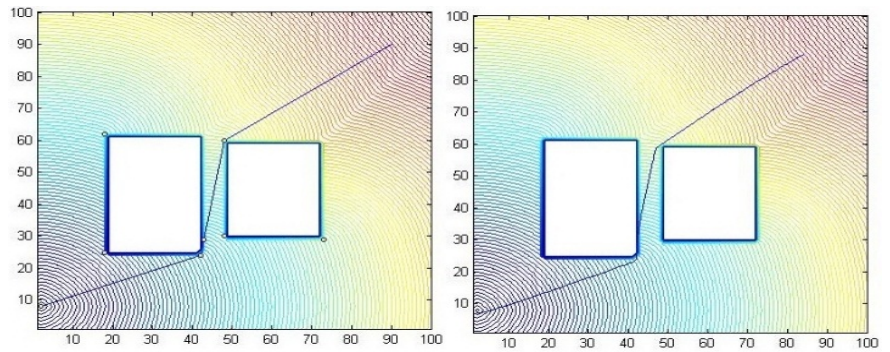

Fig. 5 Shortest paths of map1 produced by FFMM (left) and FMM (right)
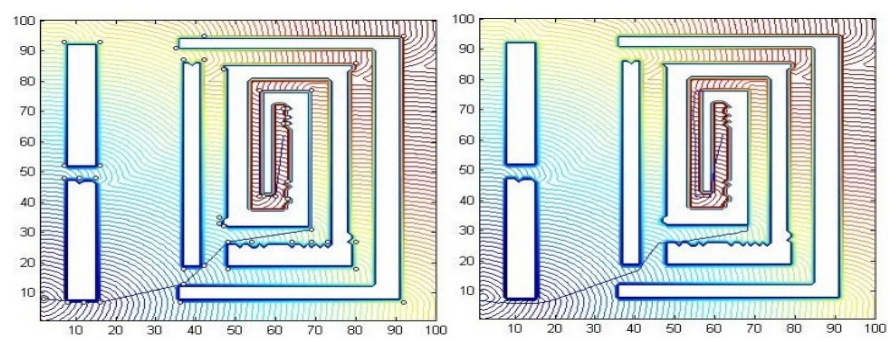

Fig. 6 Shortest paths of map2 produced by FFMM (left) and FMM (right)
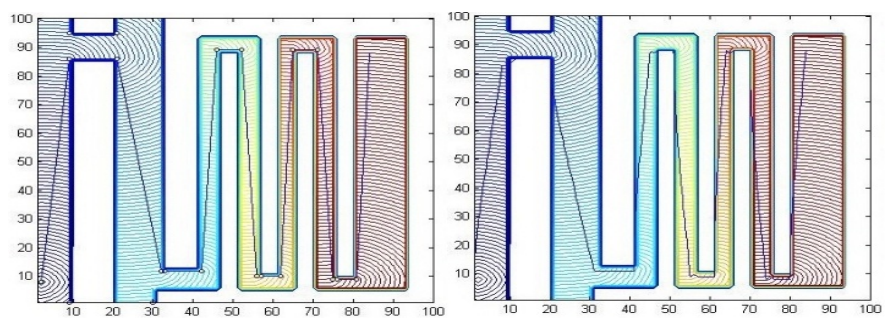

Fig. 7 Shortest paths of map3 produced by FFMM (left) and FMM (right)
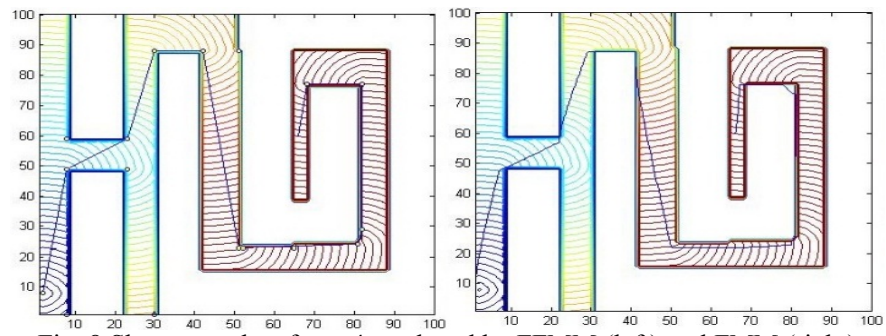

Fig. 8 Shortest paths of map4 produced by FFMM (left) and FMM (right)
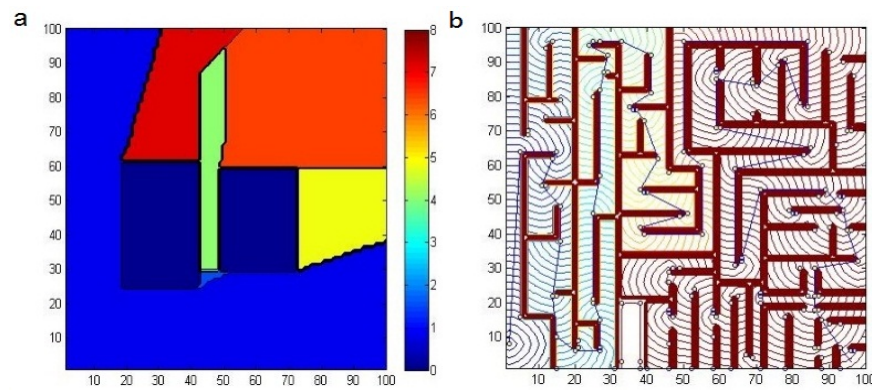

Fig. 9 FFMM TP region diagrams of map 1 (a) and FFMM shortest path of a complex maze (b)

\section{B. Dynamic path planning for pursuit-evasion problem}

In this section, we shows a simulation result of dynamic path planning for pursuit-evasion problem by applying FFMM and the path recovery rules. The map size is also $100 \times 100$ grids. The speeds of the pursuer marked in blue and the evader marked in green are set the same to 1unit/s. The evader's movement was controlled by human who tried to escape from the pursuer. The result is showed in Fig. 10 with the time lapse images. At time slot 12 second, we can see part of the path is updated after the common TP because the evader is moving a little upward. Our method cannot guarantee the success of capture when both purser and evader speeds are the same because there exists no method can guarantee the success if the evader always move to the opposite position of the pursuer between an obstacle. However, our method guarantees the success if the pursuer's speed is faster because we provide the shortest chasing path in every time slot. 

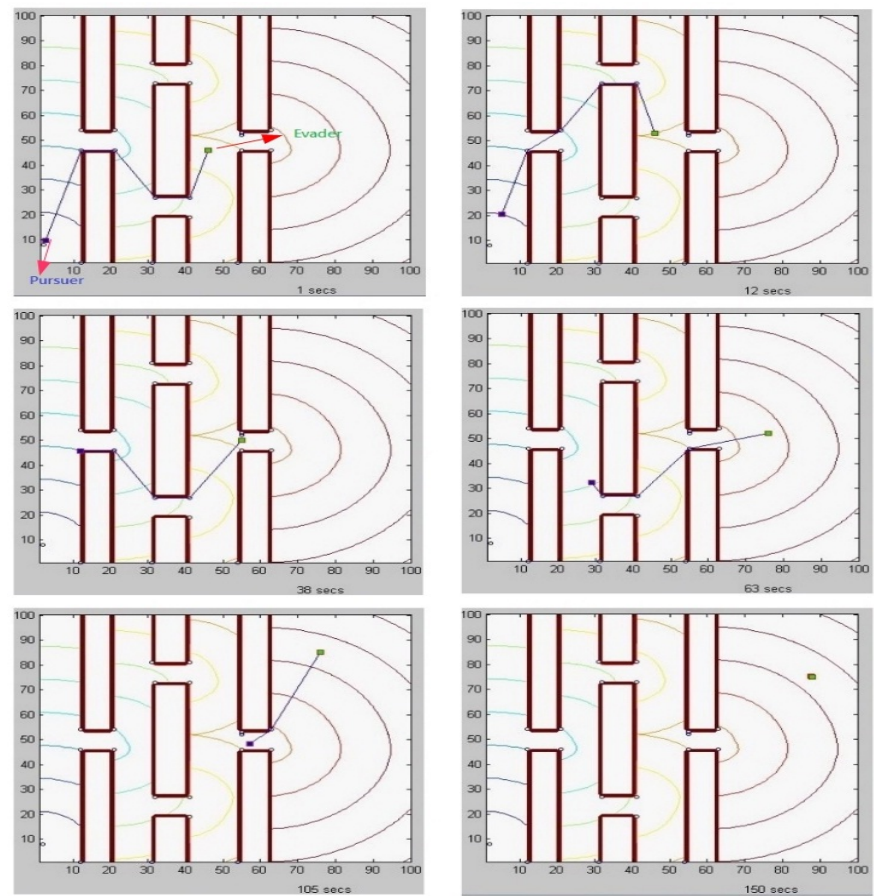

Fig. 10 Dynamic path planning for pursuit-evasion problem by FFMM. Time lapse images at 1 sec, 12 secs, 38 secs, 63 secs, 105 secs and 150secs. The pursuer is marked in blue and the evader is marked in green.

\section{CONCLUSION AND FUTURE WORKS}

Theoretically, FFMM gives better optimal path than original FMM ODE path and the experiment results show the same statement. Although the FFMM spends more time on the propagation, the tree structure result guarantees the accuracy of the generated path without concerning the errors of approximated ODE solution. In addition, the result of FFMM with the path recovery rules achieves dynamic shortest path planning in pursuit-evasion problem and improves the capture rate by providing the shortest chasing path in every time slot. Furthermore, the piecewise line function path result of FFMM is more attractive than the original $\mathrm{FMM}+\mathrm{ODE}$ in real implementation aspect because the FFMM path can provide heading directions, distances and turning angles. Those advantages make it easier to implement without trying to fix the ODE approximation error and eliminate the oscillations caused by following the point oriented path. Nevertheless, FFMM still has some issues needed to be adjusted and solved. First, when the fake TPs are not totally removed, the result may slightly worse. Fortunately, the left fake TPs are very rare and one can enlarge the range of the line examination in the algorithm to clean up them. Also, the incorrectly assigned TPs caused by linear interpolation error in the path may also slightly worse the result path but this can be solved by setting a suitable tolerance range. The other one major problem is the situation of re-doing FFMM in dynamic path planning cannot be totally avoided. However, this situation only occurs in a simple situation when the new path and the old path have no common TP. That means when the environment becomes more complicated, the chance to re-doing FFMM becomes smaller. In addition, our ongoing research with multiple pursuers can totally avoid re-doing FFMM processes. For future developments, FFMM can be boost up by running GPU calculation since all the line examinations are working on binary images. The path planning of multiple robots cooperation in pursuit-evasion problem is also a worthy trial for FFMM because a well-designed algorithm with multiple pursuers might totally avoid the situations of re-doing FFMM.

\section{REFERENCES}

[1] S. Garrido, et al., "Path Planning for Mobile Robot Navigation using Voronoi Diagram and Fast Marching," in Intelligent Robots and Systems, 2006 IEEE/RSJ International Conference, 2006, pp. 23762381.

[2] B. Mirtich and J. Canny, "Using skeletons for nonholonomic path planning among obstacles," in Robotics and Automation, 1992. Proceedings., 1992 IEEE International Conference, 1992, pp. 25332540 vol.3.

[3] J. Bruce and M. Veloso, "Real-time randomized path planning for robot navigation," in Intelligent Robots and Systems, 2002. IEEE/RSJ International Conference on, 2002, pp. 2383-2388 vol.3.

[4] S. M. LaValle and J. Kuffner, "Rapidly-Exploring Random Trees: A New Tool for Path Planning," Computer Science Dept, Iowa State University, 1998.

[5] Y. K. Hwang and N. Ahuja, "A potential field approach to path planning," Robotics and Automation, IEEE Transactions, vol. 8, pp. 2332, 1992.

[6] F. Cupertino, et al., "Fuzzy control of a mobile robot," Robotics \& Automation Magazine, IEEE, vol. 13, pp. 74-81, 2006.

[7] C. W. Warren, "Global path planning using artificial potential fields," in Robotics and Automation, 1989. Proceedings., 1989 IEEE International Conferencn, 1989, pp. 316-321 vol.1.

[8] T. Akamatsu, et al., "Real time path planning based on the potential method for an autonomous mobile overhead traveling crane," in Industrial Electronics, 2004 IEEE International Symposium, 2004, pp. 699-704 vol. 1.

[9] H. Juidette and H. Youlal, "Fuzzy dynamic path planning using genetic algorithms," Electronics Letters, vol. 36, pp. 374-376, 2000.

[10] A. A. Masoud, "A boundary value problem formulation of pursuit evasion in a known stationary environment: a potential field approach," in Robotics and Automation, 1995. Proceedings., 1995 IEEE International Conference, 1995, pp. 2734-2739 vol.3.

[11] W. Cheung and W. Evans, "Pursuit-Evasion Voronoi Diagrams in ell_1," in Voronoi Diagrams in Science and Engineering, 2007. ISVD '07. 4th International Symposium, 2007, pp. 58-65.

[12] S. Osher and J. A. Sethian, "Fronts propagating with curvaturedependent speed: algorithms based on Hamilton-Jacobi formulations," J. Comput. Phys., vol. 79, pp. 12-49, 1988.

[13] M. S. Hassouna, A. E. Abdel-Hakim and A. A. Farag., "PDE-Based Robust Robotic Navigation," presented at the Proceedings of the 2nd Canadian conference on Computer and Robot Vision, 2005.

[14] M. S. Hassouna, A. E. Abdel-Hakim and A. A. Farag., "Robust robotic path planning using level sets," in Image Processing, 2005. ICIP 2005. IEEE International Conference, 2005, pp. III-473-6.

[15] M. S. Hassouna, A. E. Abdel-Hakim and A. A. Farag., "PDE-based robust robotic navigation," Image and Vision Computing, vol. 27, pp. 10-18, 2009.

[16] D. Adalsteinsson and J. A. Sethian, "A fast level set method for propagating interfaces," J. Comput. Phys., vol. 118, pp. 269-277, 1995.

[17] S. K. Godunov, "A Difference Scheme for Numerical Solution of Discontinuous Solution of Hydrodynamic Equations," Matematicheskii Sbornik, vol. 47, pp. 271-306, translated US Joint Publ. Res. Service, JPRS 7226, 1969. 1959.

[18] M. J. Berger and J. E. Oliger, "Adaptive mesh refinement for hyperbolic partial differential equations," Stanford University, 1983. 